\title{
Minichromosome Maintenance Proteins MCM-3, MCM-5, MCM-7, and Ki-67 as Proliferative Markers in Adrenocortical Tumors
}

\author{
MICHAL APOROWICZ ${ }^{1}$, PIOTR CZOPNIK ${ }^{2}$, ELIZA KUBICKA ${ }^{3}$, ALEKSANDRA PIOTROWSKA ${ }^{4}$, \\ PIOTR DZIEGIEL ${ }^{4}$, MAREK BOLANOWSKI $^{3}$ and PAWEL DOMOSLAWSKI ${ }^{2}$ \\ ${ }^{1}$ Department and Division of Surgical Didactics, Wroclaw Medical University, Wroclaw, Poland; \\ ${ }^{2}$ Department of General, Minimally Invasive and Endocrine Surgery, \\ Wroclaw Medical University, Wroclaw, Poland; \\ ${ }^{3}$ Department and Clinic of Endocrinology, Diabetology and Isotope Therapy, \\ Wroclaw Medical University, Wroclaw, Poland; \\ ${ }^{4}$ Division of Histology and Embryology, Department of Human Morphology and Embryology, \\ Wroclaw Medical University, Wroclaw, Poland
}

\begin{abstract}
Background/Aim: Morphological features, combined with Ki-67 proliferative index, remain the standard for discriminating benign and malignant adrenocortical tumors. The aim of this study was to evaluate the role of minichromosome maintenance proteins MCM-3, MCM-5, MCM-7, and Ki-67 as proliferative markers in adrenocortical tumors. Materials and Methods: Specimens of 81 adrenocortical adenomas and 3 adrenocortical carcinomas were stained with antibodies against MCM-3, 5, 7 and Ki-67. Results: Malignant tumors were characterized by a greater size ( $p=0.017)$, volume $(p=0.017)$, and higher levels of Ki-67 $(p=0.005), M C M-3$ ( $p=0.005), M C M-7(p=0.008)$, but not MCM-5 ( $p=0.069)$. The markers' levels were independent from the tumors' size and volume, the patient's age and hormonal status. ROC curves showed Ki-67 (AUC 0.984), MCM-3 (AUC 0.984), and MCM-7 (AUC 0.950), but not MCM-5 (AUC $0.820)$ to be reliable markers. Conclusion: Ki-67, MCM-3, and MCM-7, but not MCM-5 are reliable proliferative and diagnostic markers in discerning benign and malignant adrenocortical tumors.
\end{abstract}

This article is freely accessible online.

Correspondence to: Michal Aporowicz, Department and Division of Surgical Didactics, Wroclaw Medical University, Wroclaw, Poland. Tel: +48 717842752, e-mail: micapo@interia.pl; ORCID: 0000-0002-0305-152X

Key Words: Adrenocortical adenoma, adrenocortical carcinoma, Ki67, MCM-3, MCM-5, MCM-7.
Adrenal gland tumors (AGTs) are relatively common and constitute $5-9 \%$ of all human tumors. The greater accessibility to diagnostic imaging in recent years, especially ultrasound (US) and computed tomography (CT), has revealed that the rate of AGTs is significantly higher than previously reported. The prevalence of incidentally detected adrenal mass (socalled incidentaloma) is greater with age and ranges from $0.2 \%$ (20-29 years old) to $3 \%$ (over 50 years of age) and even up to $7 \%$ (over 70 years of age) $(1,2)$. The mean value for the general population is about $2-4 \%(3,4)$. An incidentaloma is typically detected in the right adrenal gland, between the 5th and 7 th decade of life (mean age 55 years). AGTs are found on average in $1-8.9 \%$ (mean $2.3 \%$ ) of autopsies, and even in as high as $15 \%$ according to some authors $(2,4)$. Apart from an incidentaloma, adrenal tumors may present symptoms, either of hormonal excess or a mass effect (5).

Following the finding of an AGT, steps are taken to determine its origin (cortical/medullar) and character (benign/malignant) $(1,3,6)$. Most AGTs are of cortical origin and benign, adrenocortical adenoma (ACA) is the most frequent diagnosis (70-94\%) $(2,5)$. The majority of ACAs do not display hormonal activity. The most common functioning ACA is aldosterone-producing adenoma (APA), followed by cortisol-producing adenoma (CPA) (7). A malignant AGT can be either a primary adrenal lesion or a metastasis (3). Primary malignancies consist of cortical (adrenocortical carcinoma ACC) and medullar lesions (malignant pheochromocytoma about $10 \%(2.5-26 \%)$ of all pheochromocytomas) (6). Metastatic AGTs vary in origin, including lung, renal, breast, gastrointestinal (gastric, colorectal), hepatocellular carcinoma and melanoma $(1,3,5,8)$. The presence of an extra-adrenal primary malignancy increases significantly the odds of an 
AGT being malignant from $0.1 \%$ to $50-75 \%$. Adrenal incidentaloma may be a sole manifestation of a previously undiagnosed cancer in $0.2 \%$ of cases (2).

In contrast to frequently occurring ACAs, adrenocortical carcinoma (ACC) is a very rare neoplasm, with an incidence of $0.5-2 / \mathrm{million} / \mathrm{year}$, accounting for $3 \%$ of all endocrine neoplasms and $0.05-0.2 \%$ of all cancers $(2-5,9)$. It is most commonly detected in the 5 th decade in women $(5: 1 \mathrm{vs}$. men). ACC may be found accidentally (constituting $0-16 \%$, mean $4.4 \%$ of incidentalomas) or due to local clinical symptoms (combined: $40 \%$ ACCs). However, $50-60 \%$ of ACCs present hormone excess, mostly rapidly increasing hypercortisolemia (Cushing's syndrome mixed with virilization $-35 \%$, pure Cushing's syndrome $-30 \%$ ), less often pure virilization (20\%), hyperestrogenism $(10 \%)$ or Conn's syndrome (the rarest) (9). ACC is often an aggressive neoplasm, associated with a poor prognosis. Due to the lack of early symptoms, it is usually diagnosed at an advanced stage $(2,3,5,9)$.

The minichromosome maintenance (MCM) proteins were described over two decades ago. In total, there are 10 proteins in this group, named MCM1-10. MCM proteins 29 possess the characteristic ATPase domain (MCM box) and are therefore a part of the wider AAA+ group (ATPases Associated with diverse cellular Activities). MCM proteins 1 and 10 play similar roles, i.e. they are involved in DNA replication. However, they do not possess such ATPase domain. MCM 2-9 are very conservative proteins that are expressed in all eukaryotic organisms. The main function of MCM proteins is their involvement in the initiation and elongation of DNA replication, along with other factors (10, 11). They are essential in the formation of the pre-replicative complex (preRC), the replication fork and the initial steps of DNA synthesis $(11,12)$. MCM 2-7 form a hexameric, ringshaped complex that acts as one of the pre-replication factors and associates with the chromatin and the proteins of the origin recognition complex (ORC; including Cdt6 and Cdt1.15) at the M-G1 transition $(10,11)$. The MCM 2-7 complex is a non-active DNA helicase, consisting of a catalytic center (the core being formed by MCM 4,6 and 7) and regulatory subunits (MCM 2, 3 and 5). By the activity of other proteins (cdc7/dbf4 and cyclin E/cyclin-dependent kinase 2), the MCM2-7 complex becomes an active DNA helicase at the G1-S transition, enabling recruitment of additional factors to pre-RCs, the unwinding of DNA from its supercoiled state, the formation of replication forks and further DNA replication (11-13). Moreover, MCM proteins interact with many other cellular proteins, and are, therefore, involved in cellular processes such as: chromatin remodeling, maintenance of genome integrity, prevention of re-replication once per cell cycle, DNA repair, DNA transcription, RNA processing, ribosome biogenesis and cellcycle regulation $(10,11)$.
MCM proteins are vital for the process of DNA replication and are often called replication-licensing proteins. Cellular levels of MCMs depend on the cell's status, being high in proliferating cells and much lower in quiescent, differentiated or senescent cells. This makes MCMs useful markers of cell proliferation. As increased levels of MCMs are observed in dysplastic and neoplastic cells, evaluation of such levels helps detecting various precancerous states, preinvasive and invasive neoplasms. During the last decade, the assessment of MCM expression levels has been extensively investigated in diverse human malignancies, confirming their usefulness as diagnostic and prognostic factors. Numerous studies point out that MCM are more specific and sensitive than conventional proliferative markers such as $\mathrm{Ki}-67$ and PCNA. PCNA turns out to detect not only proliferating, but also quiescent cells, while Ki-67 plays an important role in ribosomic biosynthesis, but does not act as a main proliferative factor $(11,12)$.

This study evaluated the levels of three MCM proteins MCM-3, 5, and 7, as well as Ki-67 in adrenocortical tumors (ACAs and ACCs). To the best of the authors' knowledge, this is the first study concerning these molecules in AGTs. MCM-3, apart from MCM-2, is the only MCM family member that possesses an NLS domain indicating nuclear location. MCM-3 phosphorylated by cyclin B/CDK1 plays a regulatory role in MCM2-7 complex, while phosphorylation by cyclin $\mathrm{E} / \mathrm{CDK} 2$ triggers the $\mathrm{S}$ phase checkpoint activation $(11,13)$. MCM-5, in cooperation with cyclin E, associates with the centrosome and regulates its duplication (13). MCM-7 is also located within the cell nucleus. It interacts with many molecules involved in cell-cycle regulation, including $\mathrm{pRb}$, Mat-1 and FLH. It also participates in mRNA transcription and DNA damage regulation. Similar to MCM3, MCM-7 can be phosphorylated by cyclin E/CDK2 or cyclin $\mathrm{B} / \mathrm{CDK} 1$. Present in excess, phosphorylated MCM-7 blocks $\mathrm{S}$ phase entry. The phosphorylation of MCM-7 is essential for a proper mitotic exit $(11,13)$.

\section{Materials and Methods}

Patients, clinical data and specimens. All patients included in this study were operated on in the 1st Department and Clinic of General, Gastroenterological and Endocrine Surgery, Wroclaw Medical University (WMU). This study has been approved by the Bioethics Committee of the Wroclaw Medical University (opinion $\mathrm{nr} \mathrm{KB}$ 151/2016). This is a retrospective study, conducted on already available biological material, therefore formal consent was not necessary.

A database was created containing personal data, sex, age, indication for surgery, laterality of the tumor and the dimensions of the tumor for each case. Based on the size of a tumor, the volume was calculated, assuming an elliptical shape. Each patient was hospitalized in an endocrinology ward for comprehensive evaluation of their hormonal status and establishing/confirming an indication for surgery prior to admittance to our department. The vast majority 
of patients were referred to our clinic by the Department and Clinic of Endocrinology, Diabetology and Isotope Therapy, of WMU. Based on discharge papers, the hormonal status was investigated and added into the database (serum cortisol profile, free cortisol excretion in 24-h urine collection, aldosterone, and renin plasma activity - both in resting and supine position, and aldosterone excretion in 24-h urine collection). Catecholamines and derivatives, as well as androgens' metabolism markers were out of our interest; there were no androgen-producing ACAs in our observations. Formalin-fixed paraffin-embedded specimens of the respective AGTs were retrieved from the archive of the Department and Division of Pathomorphology, WMU, and passed down to Division of Histology and Embryology, Department of Human Morphology and Embryology, WMU, for laboratory processing. Four specimens of ACA (out of 85) were found severely damaged and therefore excluded from the examination.

Tissue microarrays (TMA), immunohistochemistry (IHC) and evaluation. The material for the study consisted of 81 archived paraffin embedded samples of ACA and 3 of ACC. 7- $\mu$ m thick paraffin sections were stained with hematoxylin and eosin, then scanned utilizing the histologic scanner Pannoramic MIDI (3DHistech, Budapest, Hungary) under 20× magnification to create virtual slides. The scans were examined by two independent pathologists and representative spots were selected to create microarrays ( 3 spots per block, $1.5 \mathrm{~mm}$ diameter each). Tissue microarrays (TMAs) were created by using the automatic system TMA Grand Master (3DHistech)

Immunohistochemical reactions were performed by using the Autostainer Link 48 (Dako, Glostrup, Denmark) on 4- $\mu$ m paraffin sections obtained from the TMA blocks. To deparaffinize, rehydrate and unmask the antigens, the sections were boiled in Target Retrieval Solution High pH (for MCM-3, MCM-5, MCM-7 antibodies) or in Target Retrieval Solution Low $\mathrm{pH}$ (for Ki-67 antibody) using PTLink $\left(97^{\circ} \mathrm{C}, 20 \mathrm{~min}\right)$. All IHC reactions were performed using the Dako EnVision FLEX+, Mouse, High pH (Link) (Dako, cat. number K8002) visualization system. Firstly, endogenous peroxidase was blocked using EnVision FLEX Peroxidase-Blocking Reagent for $5 \mathrm{~min}$. Subsequently, the slides were incubated with the primary antibodies: Ki-67 (MIB-1 clone, ready-to-use, Dako, cat. number IR626), MCM-3 (101 clone, 1:50, Dako, cat. number M7263), MCM-5 (1:100, Santa Cruz Biotechnology, (Dallas, TX, USA) cat. number sc-165994), and MCM-7 (1:50, Santa Cruz Biotechnology, cat. number sc-9966) for $20 \mathrm{~min}$. Afterwards, the secondary antibodies conjugated with horseradish peroxidase (HRP) were applied (EnVision FLEX /HRP) for $20 \mathrm{~min}$. Finally, a substrate for HRP (3,3'-diaminobenzidine (DAB)) was added to a 10-min incubation. All the sections were counterstained with EnVision FLEX Hematoxylin (Dako) for 5 min. The slides were washed in distilled water, then dehydrated using graded ethanol solutions $(70 \%, 96 \%, 99.8 \%)$ and xylene. Subsequently, the preparations were mounted in SUB-X Mounting Medium (Dako) using a Coverslipper (Dako).

The reactions were evaluated using an Olympus BX40 microscope, $400 \times$ magnification, and the Cell^ ${ }^{\wedge}$ imaging system (Olympus). The percentages of positive IHC reactions were calculated and added into the database.

Statistical analysis. Qualitative data were presented as percentages, and as mean \pm standard deviation. The Shapiro-Wilk test was used to
Table I. Demographic data and markers' levels in ACA and ACC groups.

\begin{tabular}{lcccl}
\hline & ACA & ACC & All & $p$-Value \\
\hline Gender & $66 \mathrm{~F} / 15 \mathrm{M}$ & $2 \mathrm{~F} / 1 \mathrm{M}$ & $68 \mathrm{~F} / 16 \mathrm{M}$ & 0.521 \\
Age & $56.7 \pm 9.8$ & $68.0 \pm 14.1$ & $57.0 \pm 10.1$ & 0.151 \\
Size & $3.76 \pm 1.76$ & $9 \pm 1.41$ & $3.89 \pm 1.93$ & $0.017^{*}$ \\
Volume & $33.2 \pm 45.9$ & $261.4 \pm 15.2$ & $38.7 \pm 57.4$ & $0.017^{*}$ \\
Ki-67 & $4.80 \pm 2.70 \%$ & $18.66 \pm 10.29 \%$ & & $0.005^{*}$ \\
MCM-3 & $7.50 \pm 5.43 \%$ & $28.54 \pm 10.72 \%$ & & $0.005^{*}$ \\
MCM-5 & $4.84 \pm 2.64 \%$ & $8.79 \pm 4.32 \%$ & & 0.069 \\
MCM-7 & $11.48 \pm 7.01 \%$ & $38.60 \pm 23.73 \%$ & & $0.008^{*}$ \\
\hline
\end{tabular}

*Statistically significant.

determine the normality of the distribution. To evaluate correlations, the Spearman Rho test was used. For the comparison of qualitative data chi-square test was used. For the comparison of quantitative data within 2 groups, the $t$-test was used for normal distribution, and the Mann-Whitney $U$-test in other cases. For the comparison of the quantitative data within $>2$ groups, the analysis of variance (ANOVA) was used for normal distribution, and the Kruskal-Wallis test in other cases. All tests were 2-tailed (except for the ShapiroWilk test). The threshold of statistical significance was set at 0.05 .

\section{Results}

In total, 84 patients $-81(96.4 \%)$ diagnosed with ACA and $3(3.6 \%)$ with ACC - were included in this study. There were 68 females $(81.0 \%)$ and 16 males $(19.0 \%), 57.0 \pm 10.1$ years. The mean tumor size was $3.89 \pm 1.93 \mathrm{~cm}$ and the mean calculated tumor volume was $38.7 \pm 57.4 \mathrm{~cm}^{3}$. The details for each group are presented in Table I. There were no significant differences between the ACA and the ACC patients in terms of sex and age. However, malignant tumors in the ACC group had a greater size $(p=0.017)$ and volume $(p=0.017)$ than those in the ACA group. Similarly, malignant tumors in the ACC group were characterized by higher levels of Ki-67 $(p=0.005)$, MCM-3 $(p=0.005)$, and MCM-7 $(p=0.008)$ than those in the ACA group. There was no difference between the ACA and the ACC groups in the level of MCM-5 $(p=0.069)$. The specimens with the lowest and highest levels of the investigated markers are presented in Figure 1.

Among the ACA group, 55 patients (67.9\%) were diagnosed with non-functioning ACA (NFA), 15 (18.5\%) had aldosterone-producing adenoma (APA), and 11 (13.6\%) had cortisol-producing adenoma (CPA). The details of each group are presented in Table II. Females were dominant in each group, although not equally: they constituted $100 \%$ of patients with CPA, $83.6 \%$ patients with NFA, and $60.0 \%$ of patients with APA. There were statistically significant differences between these groups $(p=0.027)$; females were less dominant in the APA group compared to the NFA group 

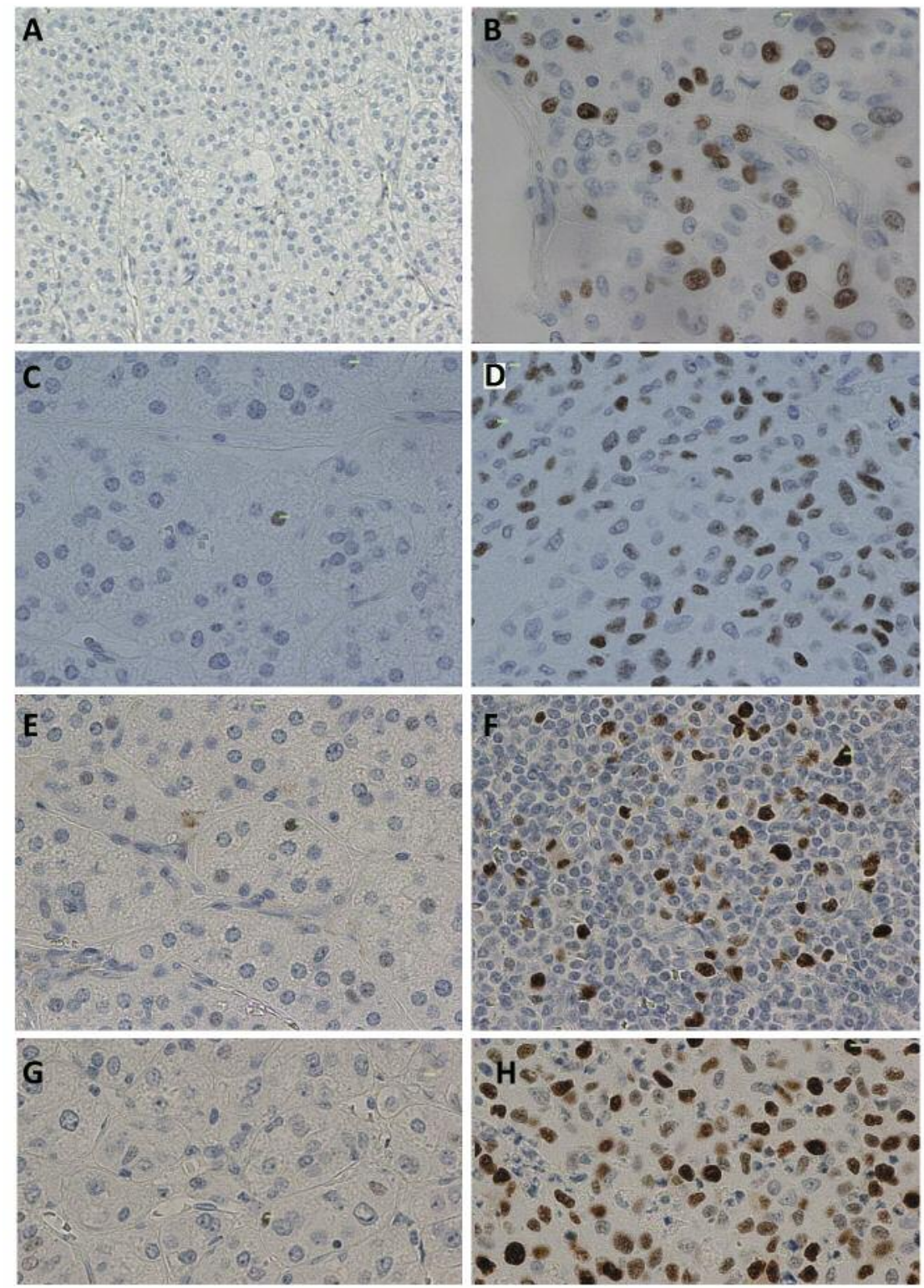

Figure 1. Specimens with the lowest and highest values of the investigated markers. A: ACA, Ki-67:0\%, 100x. B: ACC, Ki-67: 29.9\%, 400x. C: ACA, MCM-3: 1.6\%, 400x. D: ACC, MCM-3: 40.6\%, 400x. E: ACA, MCM-5: 1.0\%, 400x. F: ACC, MCM-5: 13.5\%, 400x. G: ACA, MCM-7: $1.1 \%, 400 \times . H: A C C, M C M-7: 64.3 \%, 400 \times$.

$(p=0.048)$ and the CPA group $(p=0.017)$. There were also differences in age between these groups $(p=0.004)$. Patients with NFA were older than those with APA $(p=0.030)$ and CPA $(p=0.047)$, while no differences between the APA and the CPA groups were observed $(p=0.930)$. When APA and CPA patients were combined into a single group, the difference between NFA and functioning adenomas was even more significant $(p=0.004)$.

There were significant differences in tumor size between groups $(p=0.009)$. NFAs had a significantly greater size than APAs $(p=0.007)$, but not CPAs $(p=0.084)$. APAs and ACAs did not differ in size $(p=0.072)$. The combined group of functioning adenomas had a statistically smaller size than the NFAs $(p=0.003)$. Similar differences were found in terms of calculated volume $(p=0.004)$. NFAs had significantly greater volume than APAs $(p=0.002)$, but CPAs $(p=0.057)$, as well as APAs and CPAs did not differ in volume $(p=0.176)$. The combined group of functioning adenomas had a statistically smaller volume than the NFAs $(p=0.001)$.

There were no differences in the markers' levels between the NFAs, APAs and CPAs (Ki-67, $p=0.161$; MCM-3, $p=0.810 ;$ MCM-5, $p=0.117$; MCM-7, $p=0.639)$. Besides, 
Table II. Demographic data and markers' levels in NFA, APA and CPA groups.

\begin{tabular}{|c|c|c|c|c|}
\hline & NFA & APA & CPA & $p$-Value \\
\hline Gender & 46 F/9 M $(83.6 / 16.4 \%)$ & $9 \mathrm{~F} / 6 \mathrm{M}(60.0 / 40.0 \%)$ & $11 \mathrm{~F} / 0 \mathrm{M}(100.0 / 0.0 \%)$ & $\begin{array}{c}0.027^{*} ; \mathrm{N} \text { vs. A } 0.048^{*} \\
\mathrm{~N} v s . \mathrm{C} 0.149 ; \text { A } v s . \mathrm{C} 0.017^{*}\end{array}$ \\
\hline Age & $59.1 \pm 8.2$ & $51.3 \pm 12.0$ & $51.7 \pm 10.5$ & $\begin{array}{c}0.004^{*} ; \mathrm{N} v s . \text { A } 0.030^{*} \\
\mathrm{~N} v s . \mathrm{C} 0.047^{*} ; \mathrm{A} v s . \mathrm{C} 0.930 \\
\mathrm{~N} v s . \mathrm{A}+\mathrm{C} 0.004^{*}\end{array}$ \\
\hline Size & $4.1 \pm 1.6$ & $2.9 \pm 2.3$ & $3.3 \pm 1.3$ & $\begin{array}{c}0.009^{*} ; \mathrm{N} v s . \mathrm{A} 0.007^{*} \\
\mathrm{~N} v s . \mathrm{C} 0.084 ; \mathrm{A} v s . \mathrm{C} 0.072 \\
\mathrm{~N} v s . \mathrm{A}+\mathrm{C} 0.003^{*}\end{array}$ \\
\hline Volume & $38.6 \pm 42.1$ & $26.4 \pm 68.1$ & $15.5 \pm 13.1$ & $\begin{array}{c}0.004^{*} ; \mathrm{N} v s . \mathrm{A} 0.002^{*} \\
\mathrm{~N} v s . \mathrm{C} 0.057 ; \mathrm{A} v s . \mathrm{C} 0.176 \\
\mathrm{~N} v s . \mathrm{A}+\mathrm{C} 0.001^{*}\end{array}$ \\
\hline Ki-67 & $4.36 \pm 2.08 \%$ & $6.07 \pm 4.47 \%$ & $5.29 \pm 1.77 \%$ & $0.161 ; \mathrm{N} v s . \mathrm{A}+\mathrm{C} 0.057$ \\
\hline MCM-3 & $7.19 \pm 4.54 \%$ & $8.44 \pm 8.83 \%$ & $7.75 \pm 3.58 \%$ & $0.810 ; \mathrm{N} v s . \mathrm{A}+\mathrm{C} 0.739$ \\
\hline MCM-5 & $4.66 \pm 2.61 \%$ & $4.48 \pm 2.67 \%$ & $6.25 \pm 2.57 \%$ & $0.117 ; \mathrm{N} v s . \mathrm{A}+\mathrm{C} 0.331$ \\
\hline MCM-7 & $11.48 \pm 7.10 \%$ & $12.64 \pm 8.47 \%$ & $9.90 \pm 3.99 \%$ & $0.639 ; \mathrm{N} v s . \mathrm{A}+\mathrm{C} 0.944$ \\
\hline
\end{tabular}

*Statistically significant.

after combining APAs and CPAs into a single group, there were no differences in terms of markers' levels between the NFAs and the functioning adenomas (Ki-67, $p=0.057$; MCM-3, $p=0.739$; MCM-5, $p=0.331$; MCM-7, $p=0.944)$.

Reciprocal correlations between the markers' levels are presented in Table III. Each marker's level positively correlated with one another. The correlation was strongest $(\mathrm{r} \approx 0.6-0.7)$ between Ki-67 and MCM-3 ( $\mathrm{r}=0.61, p<0.001)$, $\mathrm{Ki}-67$ and MCM-7 ( $\mathrm{r}=0.59, p<0.001)$, and MCM-3 and MCM-7 $(\mathrm{r}=0.74, p<0.001)$. The correlations between the levels of MCM-5 and other markers were also positive and significant, but weaker $(\mathrm{r} \approx 0.3-0.4)$. This reflects the fact that the levels of MCM-5 did not differ between ACAs and ACCs, while these of other markers did.

Table IV presents the correlations between the markers' levels and the tumors' size and volume, as well as the age of patients. No significant correlation was found between any marker's level and the greatest dimension of the tumor, both in the ACA subgroup and in all the tumors combined. An analysis of ACCs alone was not possible, since only 2 values were available (1 ACC was inoperative and only a surgical biopsy was taken, therefore we could not measure the tumors' size and volume; calculations based on preoperative imaging studies were also impossible). Similarly, there were no significant correlations between any marker's level and either the calculated volume of the tumor or the patient's age, both in the ACA subgroup and in all the tumors combined. Interestingly, there was a significant, positive but weak correlation between the patient's age and the tumor volume in the combined group of ACAs and ACCs ( $\mathrm{r}=0.245, p=0.025)$.

Table V presents the correlations between the markers' and the hormones' levels in all the ACAs combined, as well as limited to solely APAs or CPAs. In general, the markers' levels
Table III. Reciprocal correlations between markers' levels.

\begin{tabular}{lccc}
\hline & MCM-3 & MCM-5 & MCM-7 \\
\hline Ki-67 & $\mathrm{r}=0.61$ & $\mathrm{r}=0.42$ & $\mathrm{r}=0.59$ \\
& $p<0.001^{*}$ & $p<0.001^{*}$ & $p<0.001^{*}$ \\
MCM-3 & $\mathrm{x}$ & $\mathrm{r}=0.39$ & $\mathrm{r}=0.74$ \\
& & $p<0.001^{*}$ & $p<0.001^{*}$ \\
MCM-5 & $\mathrm{x}$ & $\mathrm{x}$ & $\mathrm{r}=0.31$ \\
& & & $p=0.004^{*}$ \\
\hline
\end{tabular}

*Statistically significant.

did not correlate with any of hormonal parameters, neither in the ACA nor the APA/CPA groups, with 4 exceptions. In the APA group, MCM-3 levels correlated significantly, positively and strongly with resting aldosterone $(\mathrm{r}=0.829, p=0.042)$ and with aldosterone in supine position ( $\mathrm{r}=0.75, p=0.02)$. Ki-67 levels correlated significantly, positively and weakly with aldosterone in the supine position in the combined ACA group ( $\mathrm{r}=0.348, p=0.032$ ). MCM-5 levels correlated significantly, negatively and strongly with aldosterone excretion in $24-\mathrm{h}$ urine collection in the APA group ( $\mathrm{r}=-0.829, p=0.042)$.

Based on the individual values of the markers' levels in each case of ACA and ACC, receiver operating characteristic (ROC) curves were created for each evaluated marker (presented on Figure 2). Ki-67 and MCM-3, followed by MCM-7, seem to be reliable markers for discriminating between ACA and ACC (convex curves, area under curve (AUC) >0.9). MCM-5 (curve almost diagonal, AUC=0.820) with a cut-off point at $7.84 \%$ only has $51.07 \%$ sensitivity and $88.00 \%$ specificity. MCM-5 levels were not different, between ACA and ACC $(p=0.069)$. 

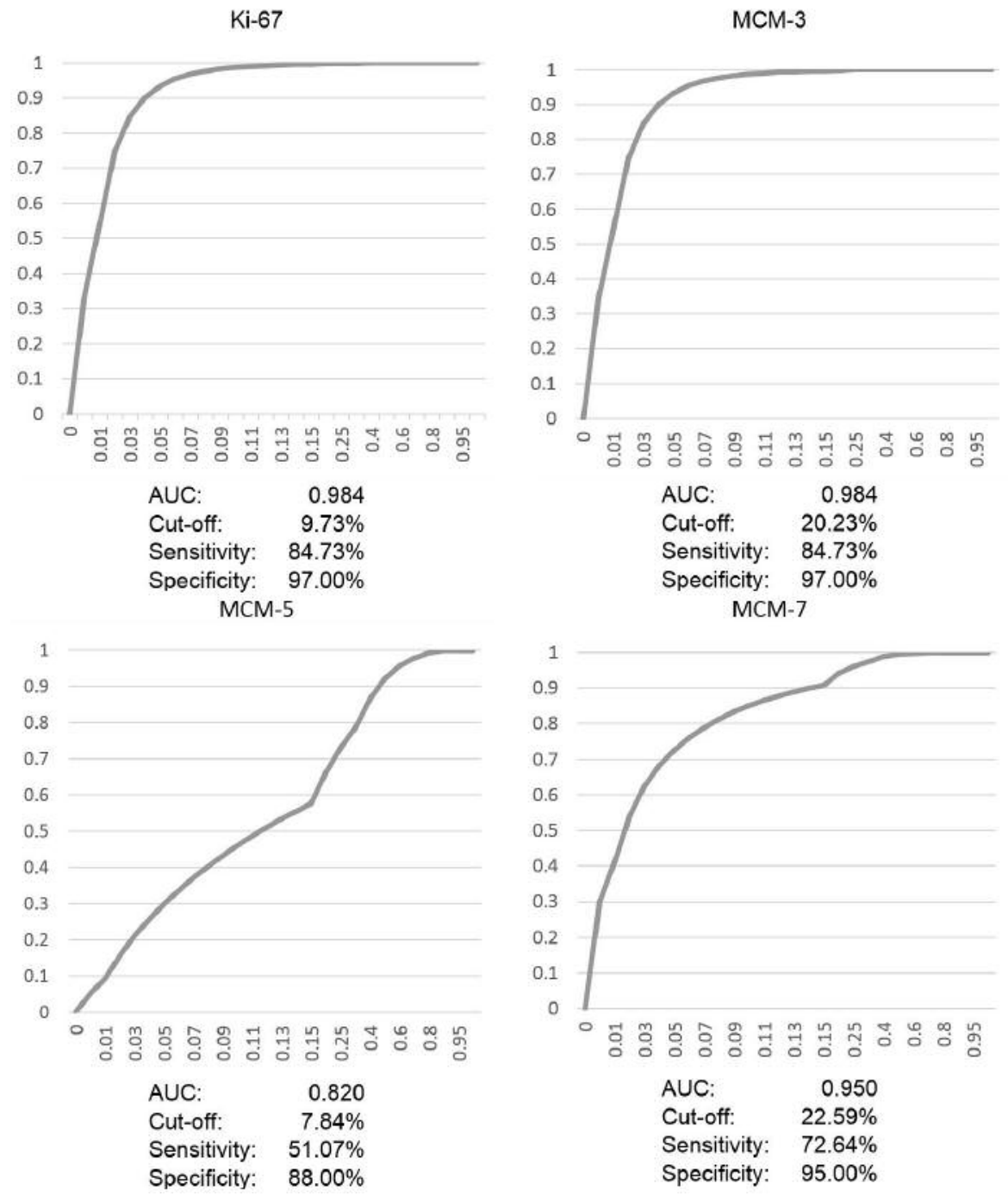

Figure 2. Receiver operating characteristic (ROC) curves created for the investigated markers.

Table IV. Correlations between markers' levels, tumor's dimension and volume and patient's age.

\begin{tabular}{lccccc}
\hline & Ki-67 & MCM-3 & MCM-5 & MCM-7 & Age \\
\hline Greatest dimension & ACA: $\mathrm{r}=-0.080$ & ACA: $\mathrm{r}=-0.043$ & ACA: $\mathrm{r}=0.090$ & ACA: $\mathrm{r}=-0.178$ & ACA: $\mathrm{r}=0.148$ \\
& $p=0.492$ & $p=0.705$ & $p=0.423$ & $p=0.111$ & $p=0.187$ \\
& all: $\mathrm{r}=-0.002$ & all: $\mathrm{r}=0.029$ & all: $\mathrm{r}=0.118$ & all: $\mathrm{r}=-0.104$ & all: $\mathrm{r}=0.204$ \\
& $p=0.982$ & $p=0.794$ & $p=0.288$ & $p=0.346$ & $p=0.064$ \\
Calculated volume & ACA: $\mathrm{r}=-0.104$ & ACA: $\mathrm{r}=-0.020$ & ACA: $\mathrm{r}=-0.171$ & ACA: $\mathrm{r}=0.192$ \\
& ACA: $\mathrm{r}=-0.114$ & $p=0.356$ & $p=0.860$ & $p=0.127$ & $p=0.085$ \\
& $p=0.312$ & all: $\mathrm{r}=-0.029$ & all: $\mathrm{r}=0.014$ & all: $\mathrm{r}=-0.098$ & all: $\mathrm{r}=0.245$ \\
all: $\mathrm{r}=-0.037$ & $p=0.795$ & $p=0.901$ & $p=0.379$ & $p=0.025^{*}$ \\
& $p=0.743$ & ACA: $\mathrm{r}=-0.034$ & ACA: $\mathrm{r}=0.026$ & ACA: $\mathrm{r}=0.116$ & X \\
& ACA: $\mathrm{r}=-0.144$ & $p=0.763$ & $p=0.818$ & $p=0.303$ & all: $\mathrm{r}=0.150$ \\
& $p=0.198$ & all: $\mathrm{r}=0.016$ & all: $\mathrm{r}=0.038$ & $p=0.174$ &
\end{tabular}

*Statistically significant. 
Table V. Correlations between markers' and hormones' levels.

\begin{tabular}{|c|c|c|c|c|}
\hline & Ki-67 & MCM-3 & MCM-5 & MCM-7 \\
\hline \multirow[t]{3}{*}{ Serum cortisol 5-6 a.m. } & $\begin{array}{c}\text { all: } r=-0.083 \\
p=0.629\end{array}$ & $\begin{array}{c}\text { all: } r=-0.119 \\
p=0.488\end{array}$ & $\begin{array}{c}\text { all: } \mathrm{r}=-0.207 \\
p=0.226\end{array}$ & $\begin{array}{c}\text { all: } r=-0.087 \\
p=0.612\end{array}$ \\
\hline & CPA: $r=0.261$ & CPA: $r=-0.232$ & CPA: $r=0.058$ & CPA: $r=-0.290$ \\
\hline & $p=0.618$ & $p=0.658$ & $p=0.913$ & $p=0.577$ \\
\hline \multirow[t]{4}{*}{ Serum cortisol 8-10 a.m. } & all: $\mathrm{r}=-0.038$ & all: $\mathrm{r}=-0.215$ & all: $\mathrm{r}=-0.236$ & all: $\mathrm{r}=-0.176$ \\
\hline & $p=0.801$ & $p=0.146$ & $p=0.110$ & $p=0.237$ \\
\hline & CPA: $r=0.036$ & CPA: $r=0.523$ & CPA: $r=-0.090$ & CPA: $r=0.162$ \\
\hline & $p=0.939$ & $p=0.229$ & $p=0.848$ & $p=0.728$ \\
\hline \multirow[t]{4}{*}{ Serum cortisol 4-8 p.m. } & all: $\mathrm{r}=-0.142$ & all: $\mathrm{r}=-0.250$ & all: $\mathrm{r}=-0.006$ & all: $\mathrm{r}=-0.168$ \\
\hline & $p=0.345$ & $p=0.094$ & $p=0.967$ & $p=0.263$ \\
\hline & CPA: $r=0.649$ & CPA: $r=0.198$ & CPA: $r=0.018$ & CPA: $r=0.198$ \\
\hline & $p=0.115$ & $p=0.670$ & $p=0.969$ & $p=0.670$ \\
\hline \multirow[t]{4}{*}{ Serum cortisol 11-12 p.m. } & all: $r=-0.067$ & all: $\mathrm{r}=-0.251$ & all: $\mathrm{r}=0.099$ & all: $\mathrm{r}=-0.080$ \\
\hline & $p=0.691$ & $p=0.128$ & $p=0.554$ & $p=0.635$ \\
\hline & CPA: $r=-0.087$ & CPA: $r=0.754$ & CPA: $r=-0.580$ & CPA: $r=0.464$ \\
\hline & $p=0.870$ & $p=0.084$ & $p=0.228$ & $p=0.354$ \\
\hline Free cortisol excretion & all: $\mathrm{r}=-0.004$ & all: $r=-0.250$ & all: $\mathrm{r}=-0.048$ & all: $r=-0.137$ \\
\hline \multirow[t]{3}{*}{ in 24 -h urine collection } & $p=0.983$ & $p=0.130$ & $p=0.774$ & $p=0.412$ \\
\hline & CPA: $r=0.541$ & CPA: $r=0.234$ & CPA: $r=-0.018$ & CPA: $r=0.018$ \\
\hline & $p=0.210$ & $p=0.613$ & $p=0.969$ & $p=0.969$ \\
\hline \multirow[t]{4}{*}{ PRA resting } & all: $\mathrm{r}=0.102$ & all: $\mathrm{r}=-0.103$ & all: $\mathrm{r}=-0.143$ & all: $\mathrm{r}=-0.211$ \\
\hline & $p=0.559$ & $p=0.556$ & $p=0.413$ & $p=0.224$ \\
\hline & APA: $r=0.154$ & APA: $r=-0.154$ & APA: $r=-0.667$ & APA: $r=-0.051$ \\
\hline & $p=0.805$ & $p=0.805$ & $p=0.219$ & $p=0.935$ \\
\hline \multirow[t]{4}{*}{ PRA supine } & all: $\mathrm{r}=0.147$ & all: $r=-0.080$ & all: $\mathrm{r}=-0.079$ & all: $\mathrm{r}=-0.191$ \\
\hline & $p=0.391$ & $p=0.641$ & $p=0.646$ & $p=0.266$ \\
\hline & APA: $r=0.058$ & APA: $r=0.464$ & APA: $r=-0.754$ & APA: $r=0.377$ \\
\hline & $p=0.913$ & $p=0.354$ & $p=0.084$ & $p=0.461$ \\
\hline \multirow[t]{4}{*}{ Aldosterone resting } & all: $\mathrm{r}=0.164$ & all: $\mathrm{r}=0.046$ & all: $r=-0.181$ & all: $\mathrm{r}=-0.081$ \\
\hline & $p=0.354$ & $p=0.798$ & $p=0.306$ & $p=0.647$ \\
\hline & APA: $r=0.486$ & APA: $r=0.829$ & APA: $r=0.600$ & APA: $r=0.657$ \\
\hline & $p=0.329$ & $p=0.042 *$ & $p=0.208$ & $p=0.156$ \\
\hline \multirow[t]{4}{*}{ Aldosterone supine } & all: $\mathrm{r}=0.348$ & all: $\mathrm{r}=0.044$ & all: $r=-0.064$ & all: $\mathrm{r}=0.072$ \\
\hline & $p=0.032 *$ & $p=0.792$ & $p=0.702$ & $p=0.668$ \\
\hline & APA: $r=0.383$ & APA: $r=0.750$ & APA: $r=0.017$ & APA: $r=0.433$ \\
\hline & $p=0.309$ & $p=0.020^{*}$ & $p=0.966$ & $p=0.244$ \\
\hline & all: $\mathrm{r}=0.096$ & all: $\mathrm{r}=0.282$ & all: $\mathrm{r}=-0.122$ & all: $\mathrm{r}=0.258$ \\
\hline \multirow{3}{*}{ in 24 -h urine collection } & $p=0.602$ & $p=0.118$ & $p=0.505$ & $p=0.154$ \\
\hline & APA: $r=-0.543$ & APA: $r=-0.429$ & APA: $r=-0.829$ & APA: $r=-0.314$ \\
\hline & $p=0.266$ & $p=0.397$ & $p=0.042 *$ & $p=0.544$ \\
\hline
\end{tabular}

*Statistically significant.

\section{Discussion}

AGTs are relatively common, with their prevalence rising along with age. Taking into account the current demographic changes in western societies, the management of AGTs will soon become a more important issue. Adrenocortical carcinoma, although rare, is associated with a very poor prognosis and few therapeutic options. Therefore, the question of discerning benign and malignant adrenocortical tumors is of great importance.

The malignant character of AGTs is mostly determined by morphologic criteria, while IHC staining plays a limited role, with the exception of $\mathrm{Ki}-67$ and the mitotic indices as diagnostic and prognostic factors. $\mathrm{Ki}-67$ is a non-histonic protein of the nuclear matrix, detected in the G1-, S-, G2- and M-phases of the cell cycle, and is, therefore, supposedly involved in DNA replication (4). A combination of Ki-67 expression and histopathological features is, to date, the best method for discerning ACCs from ACAs (4, 8, 14). Ki-67 proliferation index $(>5 \%)$ is indicative of ACC. However, ACCs with a low proliferation index may occur (5). The results of this research confirm the role of $\mathrm{Ki}-67$ in distinguishing between ACAs and ACCs $(p=0.005)$, but a higher cut-off value may be more appropriate (10\% instead of $5 \%$ ).

The presented results indicate as well that MCM-3 and MCM-7 are reliable diagnostic markers in detecting malignant 
ACTs. MCM-3 and MCM-7 levels differed significantly between ACAs and ACCs ( $p=0.005$ and 0.008 , respectively). They were independent from the tumor's size and volume, the patient's age, as well as the hormonal status (with 1 exception: MCM-3 and aldosterone). MCM-3 and MCM-7 indices ( $\geq 20 \%$ and $\geq 23 \%$, respectively) are indicative of ACC. Ki-67, MCM3 and MCM-7 levels correlated significantly, positively and strongly with one another, as shown in previous studies (11).

MCM-5 seems not to be a reliable marker for ACC. MCM5 levels did not differ significantly between ACAs and ACCs $(p=0.069)$. The ROC curve for MCM-5 was almost diagonal and with a cut-off point of $7.84 \%$ MCM-5 has only a $51.07 \%$ sensitivity in detecting ACCs (close to complete randomness). MCM-5 correlated weakly with the other investigated markers

The roles of MCM 3, 5, and 7 have never been investigated in adrenocortical tumors before. However, Szajerka et al. assessed MCM-2 expression in normal adrenal tissue, ACA and ACC. MCM-2 levels turned out to be significantly higher in ACCs when compared to ACAs and normal controls ( $p<0.05$ in both cases). A positive and significant correlation was found between Ki-67 and MCM2 levels in the malignant tumors group $(\mathrm{r}=0.136, p<0.05)$. The authors concluded that MCM-2 is a reliable marker for adrenocortical dysplasia and malignancy (4).

MCM proteins 3, 5, and 7 were previously investigated as proliferation markers in tumors of other endocrine glands, including the thyroid and the pituitary gland. Lee et al. studied MCM-3 as a potential proliferation marker in papillary thyroid carcinoma (PTC) using immunohistochemical and western blot analyses. They concluded that MCM-3 could be a more reliable prognostic marker than Ki-67 in PTC. MCM-3 levels were very low or absent in normal thyroid tissues, while they were overexpressed in PTC cells. Additionally, MCM-3 levels correlated positively and significantly with tumor size and extrathyroidal extension ( $p=0.031$ and 0.037 , respectively) (15).

Similarly, Guida et al. investigated MCM-5 and 7 in papillary and anaplastic thyroid carcinoma (ATC) using immunohistochemistry and northern blotting. The expression of both MCM proteins was negligible in normal thyroid tissue and PTC, but significantly higher in $65 \%$ of ATC cases. Moreover, the transcription rate of MCM-7 was up-regulated in ATC, since the MCM-7 promoter activity was at least 10-fold higher in ATC cells compared with normal thyroid cells (16).

Coli et al. examined MCM-7 as a prognostic marker in pituitary adenomas (PAs) and found it to be more reliable and informative than Ki-67. Among patients with invasive tumors, a high nuclear expression of MCM-7 (>15\%), but not $\mathrm{Ki}-67$, was associated with a much higher (7.7-fold) risk of recurrence or progression. The overexpression of MCM7 was a factor indicating poor clinical outcome, and might be a candidate marker for surveillance in PA patients (17).

To date, numerous studies present MCM proteins to be better proliferative and diagnostic markers for various malignancies than the classic Ki-67 and PCNA. Indeed, the potential for DNA replication seems to be dependent on the MCM complex. However, MCM proteins take part in many other cellular activities, and most of them are found in sites other than DNA synthesis. Additionally, a reduction of $90 \%$ of the cellular amount of MCM proteins seems not to hinder DNA replication or cell cycle progression; this phenomenon is called the "MCM paradox" (10). Perhaps future studies will characterize the position of MCM proteins as proliferative and diagnostic markers more comprehensively.

Moreover, our study confirmed that ACCs are significantly greater in terms of size and volume than ACAs $(p=0.017)$. This is in accordance with other investigators' observations that most ACCs are generally larger than 5-6 cm in diameter and $100 \mathrm{~g}$ in weight $(1,2,5)$. In our study, two measureable ACCs had 10 and $8 \mathrm{~cm}$ in the greatest dimension, and 272 and $250 \mathrm{~cm}^{3}$ in volume, respectively. We also detected that functioning ACAs are smaller in both size and volume ( $p=0.003$, and 0.001 , respectively), and are detected in younger patients $(p=0.004)$ than non-functioning ones. There is no consensus among other researchers about the differences in size between functioning and non-functioning ACAs. Maurea et al. found NFAs to be larger than functioning ACAs, but not significantly (18). In turn, Hara et al. stated that CPAs are larger than APAs (19). We observed a similar relation, but not significant $(p=0.072)$. Vasilev et al. observed that NFAs are smaller than CPAs, and similar in size to APAs, which is in complete opposition to our results (20). In the combined group of all the examined AGTs, the tumors of younger patients had smaller volume $(p=0.025)$.

The most important limitation of this study was the insufficient amount of ACC. However, taking into account the low prevalence of ACC, this seems to be justified.

In conclusion, Ki-67, MCM-3, and MCM-7, but not MCM-5 are reliable proliferative and diagnostic markers in discerning benign and malignant adrenocortical tumors.

\section{Conflicts of Interest}

The Authors declare that they have no conflict of interest regarding this study.

\section{Authors' Contributions}

MA, PDz, MB and PDo designed the study. MA, PC, EK and AP collected the data. MA performed statistical analysis. MA, PC, AP and $\mathrm{MB}$ prepared the manuscript. $\mathrm{MA}$ and $\mathrm{MB}$ searched the literature. MA, PDz and PDo collected funds. All authors interpreted the data and corrected the manuscript.

\section{Acknowledgements}

This study was funded by the Wroclaw Medical University (grant number ST-922). 


\section{References}

1 Bednarczuk T, Bolanowski M, Sworczak K, Górnicka B, Cieszanowski A, Otto M, Ambroziak U, Pachucki J, Kubicka E, Babińska A, Koperski Ł, Januszewicz A, Prejbisz A, Górska M, Jarząb B, Hubalewska-Dydejczyk A, Glinicki P, Ruchała M and Kasperlik-Załuska A: Adrenal incidentaloma in adults management recommendations by the Polish Society of Endocrinology. Endokrynol Pol 67: 234-258, 2016. PMID: 27082051, DOI: 10.5603/EP.a2016.0039

2 Harrison B: The indeterminate adrenal mass. Langenbecks Arch Surg 397: 147-154, 2012. PMID: 21947480, DOI: 10.1007/ s00423-011-0845-0

3 Enriquez ML, Lal P, Ziober A, Wang L, Tomaszewski JE and Bing Z: The use of immunohistochemical expression of SF-1 and EMA in distinguishing adrenocortical tumors from renal neoplasms. Appl Immunohistochem Mol Morphol 20: 141-145, 2012. PMID: 22553814, DOI: 10.1097/PAI.0b013e31823605e7

4 Szajerka A, Dziegiel P, Szajerka T, Zabel M, Winowski J and Grzebieniak Z: Immunohistochemical evaluation of metallothionein, Mcm-2 and Ki-67 antigen expression in tumors of the adrenal cortex. Anticancer Res 28: 2959-2966, 2008 PMID: 19031940

5 Tissier F: Classification of adrenal cortical tumors: What limits for the pathological approach? Best Pract Res Clin Endocrinol Metab 24: 877-885, 2010. PMID: 21115156, DOI: 10.1016/ j.beem.2010.10.011

6 Otto M: Surgical treatment of adrenal tumours. Endokrynol Pol 61: 716-722, 2010. PMID: 21104648

7 Nanba K, Tsuiki M, Sawai K, Mukai K, Nishimoto K, Usui T, Tagami T, Okuno H, Yamamoto T, Shimatsu A, Katabami T, Okumura A, Kawa G, Tanabe A and Naruse M: Histopathological Diagnosis of Primary Aldosteronism Using CYP11B2 Immunohistochemistry. J Clin Endocrinol Metab 98: 1567-1574, 2013. PMID: 23443813, DOI: 10.1210/jc.2012-3726

8 Papotti M, Duregon E, Volante M and McNicol AM: Pathology of the adrenal cortex: a reappraisal of the past 25 years focusing on adrenal cortical tumors. Endocr Pathol 25: 35-48, 2014. PMID: 24382573, DOI: 10.1007/s12022-013-9291-6

9 Nowak KM, Samsel R, Cichocki A, Ambroziak U, RoszkowskaPurska K, Lebek-Szatanska A, Koperski L, Otto M, Zgliczynski W and Papierska L: Prognostic factors in adrenocortical carcinoma: data from a large Polish series. Pol Arch Intern Med 128: 371-378, 2018. PMID: 29726479, DOI: 10.20452/ pamw.4260

10 Dubois ML, Bastin C, Levesque D and Boisvert FM: Comprehensive characterization of minichromosome maintenance complex $(\mathrm{MCM})$ protein interactions using affinity and proximity purifications coupled to mass spectrometry. J Proteome Res 15: 2924-2934, 2016. PMID: 27494197, DOI: 10.1021/acs.jproteome. $5 \mathrm{~b} 01081$

11 Nowinska K and Dziegiel P: The role of MCM proteins in cell proliferation and tumorigenesis. Postepy Hig Med Dosw (online) 64: 627-635, 2010. PMID: 21160097
12 Giaginis C, Vgenopoulou S, Vielh P and Theocharis S: MCM proteins as diagnostic and prognostic tumor markers in the clinical setting. Histol Histopathol 25: 351-370, 2010. PMID: 20054807, DOI: $10.14670 / \mathrm{HH}-25.351$

13 Wei Q, Li J, Liu T, Tong X and Ye X: Phosphorylation of Minichromosome Maintenance protein 7 (MCM7) by cyclin/cyclin-dependent kinase affects its function in cell cycle regulation. J Biol Chem 288: 19715-19725, 2013. PMID: 23720738, DOI: 10.1074/jbc.M112.449652

14 Babinska A, Sworczak K, Wisniewski P, Nalecz A and Jaskiewicz K: The role of immunohistochemistry in histopathological diagnostics of clinically "silent" incidentally detected adrenal masses. Exp Clin Endocrinol Diabetes 116: 246-251, 2008. PMID: 18393131, DOI: 10.1055/s-2007-993164

15 Lee YS, Ha SA, Kim HJ, Shin SM, Kim HK, Kim S, Kang CS, Lee KY, Hong OK, Lee SH, Kwon HS, Cha BY and Kim JW: Minichromosome maintenance protein 3 is a candidate proliferation marker in papillary thyroid carcinoma. Exp Mol Pathol 88: 138-142, 2010. PMID: 19818763, DOI: 10.1016/ j.yexmp.2009.09.015

16 Guida T, Salvatore G, Faviana P, Giannini R, Garcia-Rostan G, Provitera L, Basolo F, Fusco A, Carlomagno F and Santoro M: Mitogenic effects of the up-regulation of minichromosome maintenance proteins in anaplastic thyroid carcinoma. J Clin Endocrinol Metab 90: 4703-4709, 2005. PMID: 15899946, DOI: 10.1210/jc.2004-2459

17 Coli A, Asa SL, Fadda G, Scannone D, Chiloiro S, De Marinis L, Lauretti L, Ranelletti FO and Lauriola L: Minichromosome maintenance protein 7 as prognostic marker of tumor aggressiveness in pituitary adenoma patients. Eur J Endocrinol 174: 307-314, 2016. PMID: 26620390, DOI: 10.1530/EJE-150586

18 Maurea S, Imbriaco M, Mollica C, Pace L and Salvatore M: Quantitative imaging characterization of hypersecreting or nonhypersecreting adrenal adenomas: comparison between iodine-131 norcholesterol uptake and magnetic resonance signal intensity ratios. Nucl Med Commun 32: 535-541, 2011. PMID: 21448089, DOI: 10.1097/MNM.0b013e32834319e3

19 Hara I, Kawabata G, Hara S, Yamada Y, Tanaka K and Fujisawa M: Clinical outcomes of laparoscopic adrenalectomy according to tumor size. Int J Urol 12: 1022-1027, 2005. PMID: 16409603, DOI: $10.1111 / \mathrm{j} .1442-2042.2005 .01199 . x$

20 Vasilev V, Matrozova J, Elenkova A and Zacharieva S: Clinical characteristics and follow-up of incidentally found adrenal tumours - results from a single tertiary centre. Cent Eur J Med 9: 292-301, 2014. DOI: $10.2478 / \mathrm{s} 11536-013-0199-9$

Received January 14, 2019

Revised January 26, 2019

Accepted January 28, 2019 